\title{
Characterization of Thermally Evaporated Ag-Ge-S Thin Films
}

Fei Wang ${ }^{1,2}$, William Porter Dunn ${ }^{2}$, Mukul Jain ${ }^{2}$, Carter De Leo ${ }^{2}$, Nicholas Vicker ${ }^{2}$, Richard Savage $^{2}$, Xiaomin $\mathrm{Jin}^{2}$, Sergey Mamedov ${ }^{3}$, and Punit Boolchand ${ }^{4}$

${ }^{1}$ California State University at Long Beach, Long Beach, CA, 90840

${ }^{2}$ California Polytechnic State University, San Luis Obispo, CA, 93407

${ }^{3}$ Horiba Jobin Yvon Inc, Edison, NJ, 08820

${ }^{4}$ University of Cincinnati, Cincinnati, OH, 45221

\section{ABSTRACT}

Thin films of ternary $\left(\mathrm{GeS}_{3}\right)_{1-\mathrm{x}} \mathrm{Ag}_{\mathrm{x}}$ glasses $(\mathrm{x}=0.1$ and 0.2$)$ are characterized in this studied as potential media for information storage. The films are deposited in a vacuum thermal evaporator at 3 different evaporation angles $\left(0^{\circ}, 30^{\circ}\right.$ and $\left.45^{\circ}\right)$ and are examined by Raman spectroscopy. The Raman spectra from both normally and obliquely deposited thin film samples reveal Ge-S CS modes $\left(\sim 340 \mathrm{~cm}^{-1}\right)$, Ge-S ES $\left(\sim 360 \mathrm{~cm}^{-1}\right)$ modes and thiogermanate modes $\mathrm{Q}_{1} \sim \mathrm{Q}_{3}\left(390 \mathrm{~cm}^{-1} \sim 437 \mathrm{~cm}^{-1}\right)$. In addition, the spectra contain sharp peaks due to sulfur rings $\left(\mathrm{S}_{8}\right)$, which are observed at $218 \mathrm{~cm}^{-1}$ and $470 \mathrm{~cm}^{-1}$. The Raman line-shapes are qualitatively consistent with those observed in bulk glasses. However, the sharp peaks due to sulfur rings were not observed in bulk glasses. By comparing the CS modes at three angles, we observe that normally deposited ( 0 degree) thin films show a red-shift towards the center and a broadening in width. Film thickness of normally deposited films are significantly less when compared against obliquely deposited films.

\section{INTRODUCTION}

Chalcogenide glasses are extensively used in rewritable non-volatile memory applications. In general, information storage devices based on chalcogenide materials could be categorized into two types. One is information storage based on the phase change of chalcogenide materials between an amorphous state and a crystalline state [1]. This technique is widely used in rewritable optical data recording (e.g. RW-DVD discs). Materials used as active recording layers for this category are $\mathrm{Sb}$-Te containing alloys, with the most widely used material being the Ge-Sb-Te (GST) system [2-4].

The other type of information storage mechanism is relatively new. It is known as Programmable Metallization Cell memory (PMC). This type of memory device controls the amount of metal dopants in a solid state electrolyte using electrochemical methods [5-6]. Therefore, one can control the resistivity of the solid state electrolyte to achieve the data recording purpose. Materials used as active recording films for this category are metal doped chacogenides, such as Ag-Se, Ag-S[7], Ag-Ge-Se [5-6], Ag-Ge-S [8], Cu-S [9]etc. PMC, compared to phase change memory, has the advantages in terms of short recording time, low recording power as well as better scaling capability [5-6].

Silver doped germanium sulfide (Ag-Ge-S) films have demonstrated PMC behaviour and several successful devices have been fabricated. [7,8] To further understand these films, one needs to have a thorough knowledge of the characteristics of film, such as its molecular structure, stress and process related variations stoichiometry. 
The aim of this work is to study the structure of Ag-Ge-S thin films deposited at different deposition angles. Our films are prepared using vacuum thermal evaporation methods and they are characterized using Raman spectroscopy.

\section{EXPERIMENT}

Ternary Ag-Ge-S glasses with composition $\operatorname{Ag}_{\mathrm{x}}\left(\mathrm{Ge}_{25} \mathrm{~S}_{75}\right)_{1-\mathrm{x}}(\mathrm{x}=0.1$ and 0.2$)$ are synthesized by mixing measured amount of Ag mesh (99.999\%), Ge shots (99.999\%) and Sulfur chips $(99.999 \%)$ in a vacuumed quartz tubing. The starting materials are melted in the sealed quartz tube after placing it into a furnace and then increasing temperature slowly $\left(1^{\circ} \mathrm{C} / \mathrm{min}\right)$ to $950^{\circ} \mathrm{C}$. The melts are homogenized at $950^{\circ} \mathrm{C}$ for $2-3$ days before the temperature is reduced down to around $600^{\circ} \mathrm{C}$. Samples are watered quenched after being homogenized at $600^{\circ} \mathrm{C}$ for at least 12 hours. Bulk sample synthesis was completed at the Solid State Physics and Electronic Materials Lab at the University of Cincinnati, Ohio.

Thin films were fabricated using a Varian VE-10 vacuum evaporator. Fresh quenched samples were aged in quartz tubes for 2 months before being evaporated. Powdered bulk materials were then loaded into evaporation boats and covered using fine tungsten mesh in order to avoiding spitting during the evaporation process. Glass substrates were placed at $0^{\circ}, 30^{\circ}$ and $45^{\circ}$ locations, as shown in Figure 1.

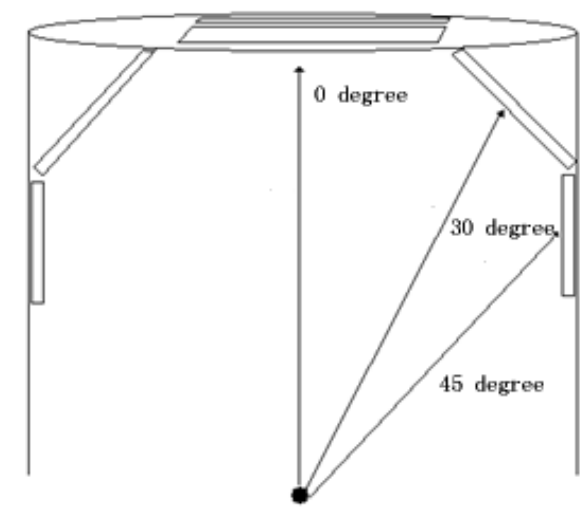

Figure 1 Substrate layout in bell jar.

The thickness of the evaporated thin films from all three angles were measured by a profiliometer. The Raman spectra of the films were obtained using a He-Ne laser (632.8 nm) focused through a $100 \mathrm{X}$ objective. The optical power at the sample was $0.6 \mathrm{~mW}$. (Raman experiments were performed at Horiba Jobin Yvon Inc., New Jersey).

\section{DISCUSSION}

\section{-Normally deposited films}

Figure 2 shows the Raman line-shapes obtained from thin-films of $\operatorname{Ag}_{\mathrm{x}}\left(\mathrm{Ge}_{25} \mathrm{~S}_{75}\right)_{1-\mathrm{x}}$ $(\mathrm{x}=10 \%)$, corresponding bulk material and bulk $\mathrm{Ge}_{25} \mathrm{~S}_{75}$, respectively $[10,11]$. The Raman results from the thin films qualitatively agreed with that of the corresponding bulk materials. This indicates the successful deposition of Ag-Ge-S thin films using thermal evaporation. In both thin films and bulk, the Raman results clearly show Corner-sharing (CS) Ge-S $\mathrm{S}_{4}$ tetrahedra ( 340 $\left.\mathrm{cm}^{-1}\right)$, Edge-sharing (ES) Ge-S $\mathrm{S}_{4}$ tetrahedra $\left(\sim 360 \mathrm{~cm}^{-1}\right)$ and thio-germanate units $\mathrm{Q}_{\mathrm{m}}, m=1,2,3$ 
$\left(\sim 370 \mathrm{~cm}^{-1}, 390 \mathrm{~cm}^{-1}\right.$ and $405 \mathrm{~cm}^{-1}$, respectively). The existence of thio-germanate units in the thin films was verified by comparison with $\mathrm{Ge}_{25} \mathrm{~S}_{75}$ [12]. The Raman of $\mathrm{Ge}_{25} \mathrm{~S}_{75}$ showed a deep valley between $370 \mathrm{~cm}^{-1}$ and $420 \mathrm{~cm}^{-1}$, while that of $\mathrm{Ag}_{\mathrm{x}}\left(\mathrm{Ge}_{25} \mathrm{~S}_{75}\right)_{1-\mathrm{x}}(\mathrm{x}=10 \%)$ thin film was flat, which indicates some modes in that region. Thio-germanate units are clear signs of Ag mingling within the molecular lattice. Here, $m$ represents the number of bridging sulfur in the units. The inset in Figure 2 gives an illustration for the $\mathrm{Q}_{3}$ units. (unit has 3 bridging sulfur and 1 sulfur attached by Ag).

Beside the similarity, there were also some discrepancies between the Raman spectra from the thin films and those from the bulk. Sharp peaks were observed at $220 \mathrm{~cm}^{-1}$ and $470 \mathrm{~cm}^{-1}$ in the thin film but not in the bulk. These peaks are known to correspond to $\mathrm{S}_{8}$ rings. This is mainly due to the low vaporization temperature of sulfur $\left(57^{\circ} \mathrm{C}\right)$. During the evaporation, sulfur is always evaporated earlier than $\mathrm{Ag}$ and Ge. This may result in a film that is richer in sulfur than its corresponding bulk.

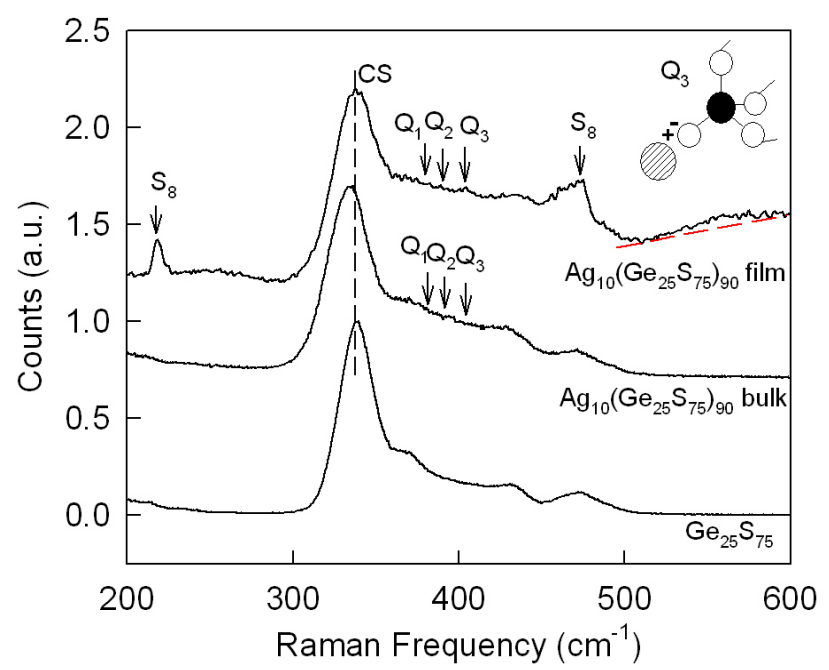

Figure 2 Raman results of $\operatorname{Ag}_{x}\left(G e_{25} S_{75}\right)_{1-x}(x=10 \%)$ thin film, $\operatorname{Ag}_{x}\left(G_{25} S_{75}\right)_{1-x}(x=10 \%)$ bulk and $\mathrm{Ge}_{25} \mathrm{~S}_{75}$ bulk $[10,11]$.

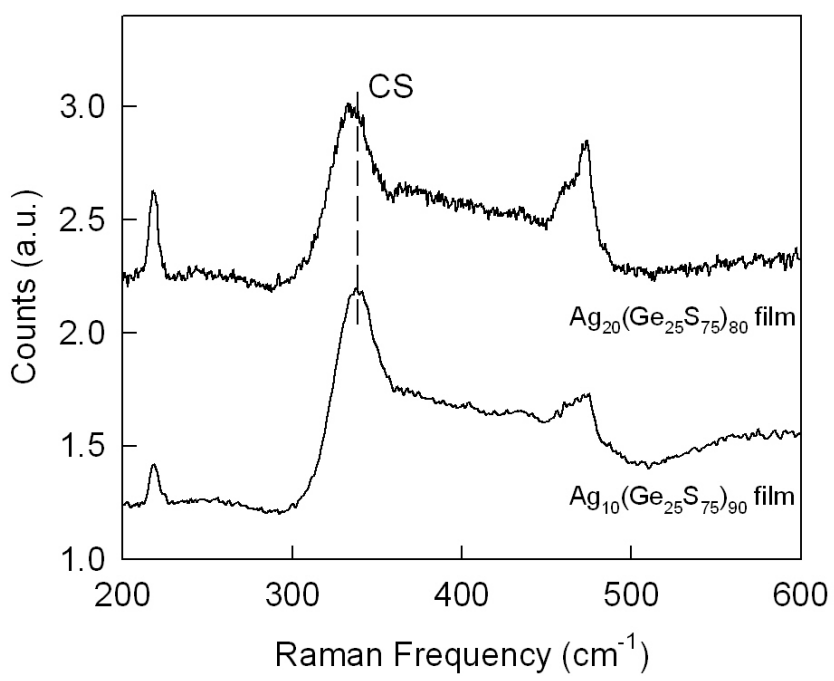

Figure 3 Raman line-shapes of thin film $\operatorname{Ag}_{x}\left(G_{25} S_{75}\right)_{1-x}(x=10 \%$ and $20 \%)$ 
Figure 3 compares the thin films of $10 \%$ and $20 \%$ of $\mathrm{Ag}_{\mathrm{x}}\left(\mathrm{Ge}_{25} \mathrm{~S}_{75}\right)_{1-\mathrm{x}}$. The CS mode of $20 \%$ film was red shifted compare to that of the $10 \%$ film. There are more than one mechanism that can cause this phenomenon. According to Y. Wang's work on Ag-Ge-Se $[13,14]$ and F. Wang's work on bulk Ag-Ge-S [11-12], after silver enters the Ge-S lattice, some atoms will attach to the Ge-S tetrahedral to form thio-germanate units, the rest will segregate onto the back bone to form $\mathrm{Ag}_{2} \mathrm{~S}$. Ag2S segregation reduces the stress of the backbone, which appears in the Raman spectra as a red shift. On the other hand, bond defects and lattice disorder will also result

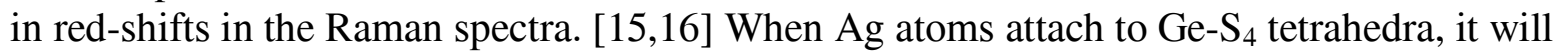
introduce more bond disorder due to the oversized Ag-ion, which will also result in red shifts in the Raman spectra. The red-shift was more significant in $\mathrm{x}=20 \%$ films due to more segregation and bond disorder because more silver atoms were involved.

\section{-Obliquely deposited thin films}

The Raman results for obliquely deposited films are shown in Figure 4. Qualitatively, the Raman results of thin films deposited at all three angles agree with each other. Peakfit (AISN Software Inc.) was utilized to de-convolute the Raman line-shapes into a combination of Gaussian peaks. The FHWM of CS modes showed a decreasing trend from 0 degrees to 45 degrees, while a red-shift in the peak center was observed (see Table 1). Line-widths of Raman scattering are well accepted as a measure of disorder in glassy lattices, which in turn results in red-shift in mode frequencies. Normal deposition (0 degrees) introduces more significant bond disorder probably because the atoms in a vapor are normally bombarding onto substrates, which will likely result in defects in the bonds.

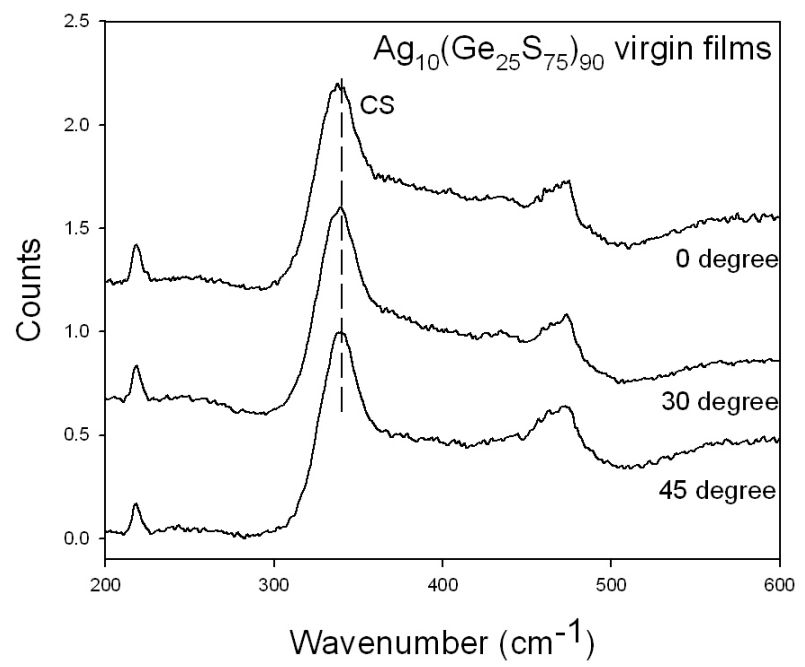

Figure 4 results from thin films deposited at 0 degree (normal deposition), 30 degree and 45 degree.

In Table 1, the thickness measurement for thin films at all three degrees from the same batch are listed (the values in Table 1 are an average from 6 films for each deposition angle). The thicknesses of normally deposited thin films are significantly smaller than those of obliquely deposited films. This could also be due to the larger bombarding energy produced during the film deposition process in a normal deposition setup. 
Table 1 Comparison of Raman spectroscopy and thickness measurements for three deposition angles.

\begin{tabular}{|l|r|r|r|r|}
\hline & CS Center $\left(\mathrm{cm}^{-1}\right)$ & CS FHWM $\left(\mathrm{cm}^{-1}\right)$ & CS AMP & Thickness $(\mathrm{nm})$ \\
\hline 0 degree & 336.13 & 28.54 & 0.86 & 192 \\
\hline 30 degree & 337.74 & 27.74 & 0.91 & 246 \\
\hline 45 degree & 337.95 & 26.22 & 0.833 & 251 \\
\hline
\end{tabular}

\section{CONCLUSIONS}

$\left(\mathrm{GeS}_{3}\right)_{1-\mathrm{x}} \mathrm{Ag}_{\mathrm{x}}(\mathrm{x}=0.1$ and 0.2$)$ thin films were fabricated using a vacuum thermal evaporation method at three different deposition angles ( 0 degrees, 30 degrees and 45 degrees). Raman spectroscopy results showed that the molecular structure of the thin films qualitatively agreed with those produced by corresponding bulk materials, with the exception of $S_{8}$ modes. This was due to the low vaporization temperature of sulfur. By comparing CS modes of thin films deposited at three angles, it was determined that normally deposited ( 0 degree $)$ films showed a red-shift in their center and a broadening in their width. Film thickness of normally deposited films was significantly smaller when compared with corresponding obliquely deposited films. The conclusion from the interpretation of these results was that normal deposition introduced atoms into the lattice with higher energy, which resulted in more bond defects.

\section{ACKNOWLEDGMENTS}

This work was sponsored by the Department of the Navy, Office of Naval Research, under Award \# N00014-06-1-1111

\section{REFERENCES}

1. S. R. Ovshingsky, Phys. Rev. Lett. 21, 1450 (1968)

2. T. Ohta, J. Optoelectron. Adv. Later. 3, 609 (2001)

3. J. Siegel, A. Schropp, J. Solis, C.N. Afonso, M. Wuttig, Appl. Phys. Lett. 84, 2250 (2004)

4. T. Ohta, E.R. Ovshynsky, in Photo-Induced Metastavility in Amorphous Semiconductors, edited by A.V. Kovobov, Wiley-VCH, Weinheim, p. 310 (2003)

5. M.N. Kozicki, M. Yun, S. J. Yang, J.P Aberouette, J.P. Bird, Superlattices and Microstructures, 27, No. 5/6 (2000)

6. M. N. Kozicki, C. Gopalan, M. Balakrishnan, M. Park, M. Mitkova, Non-Volatile Memory Technology Symposium Proceedings 15-17 (2004)

7. I. Chaitanya et al. presentation at American Physical Society March Meeting, Baltimore, MD, 2006 (unpublished)

8. Carter De Leo, Senior Project Report, California Polytechnic State University (2007)

9. Sakamoto et al. Appl. Phys. Lett. 82 (2003)

10. P. Boolchand, Fei Wang and Uday Vempati, presentation at American Physical Society March Meeting, Montreal, Canada 2004 (unpublished)

11. Fei Wang, M. Mitkova, P. Boolchand, presentation at $22^{\text {nd }}$ International Conference on Amorphous and Nanocrystalline Semiconductors, Denver, Colorado 2007 (unpublished)

12. Fei Wang, PhD Thesis, University of Cincinnati (2005)

13. Y. Wang, M. Mitkova, D.G. Georgiev, S. Mamedov, P. Boolchand, J. Phys: Condensed Matter, 15, S1573 (2003)

14. M. Mitkova, Y. Wang, P. Boolchand, Phys. Rev. Lett. 83, 3848 (1999) 
15. M. X. Gu et al. J. of Raman Spectrosc. 38, 780-788 (2007)

16. M. Stabl, L. Ticky, Solid State Science, 7, 201-207 (2005) 